Courrier du Centre International BlaisePascal

38-39 | 2017-2018

Varia

\title{
Compte rendu du Colloque « Foi et raison chez Pascal et Chestov »
}

Bernard Grasset

\section{OpenEdition}

1 Journals

Édition électronique

URL : https://journals.openedition.org/ccibp/1503

DOI : 10.4000/ccibp.1503

ISSN : 2493-7460

Éditeur

Centre international Blaise Pascal

Édition imprimée

Date de publication : 1 janvier 2019

Pagination : 191-196

ISBN : 978-2-84516-897-8

ISSN : 0249-6674

Référence électronique

Bernard Grasset, « Compte rendu du Colloque « Foi et raison chez Pascal et Chestov » », Courrier du Centre International Blaise-Pascal [En ligne], 38-39 | 2017-2018, mis en ligne le 21 juin 2021, consulté le 05 mai 2022. URL : http://journals.openedition.org/ccibp/1503; DOI : https://doi.org/10.4000/ccibp. 1503

Ce document a été généré automatiquement le 5 mai 2022.

Centre international Blaise Pascal 


\title{
Compte rendu du Colloque « Foi et raison chez Pascal et Chestov »
}

\author{
Bernard Grasset
}

1 Peu de temps après la soutenance de la thèse «Les Pensées de Pascal, une interprétation de l'Écriture » et la publication de l'essai correspondant, j'ai rencontré au Salon de la Revue à Paris, sur le stand de la Société d'Études Léon Chestov, Ramona Fotiade, la Présidente. Je lui ai dit que j'avais été sensible à l'intérêt de la lecture chestovienne de Pascal, elle m'a dit qu'il serait bon d'organiser un colloque autour de ces deux auteurs. Une quinzaine d'années plus tard, grâce au soutien bienveillant et éclairant de Gérard Ferreyrolles, était organisé, par la Société d'Études Léon Chestov en partenariat avec l'Université Paris-Sorbonne, le colloque «Foi et raison chez Pascal et Chestov ». Ce colloque international, dont j'avais proposé le thème, ainsi que le titre, qui me paraissaient le meilleur point de rencontre, de confrontation, entre les deux penseurs religieux de l'existence, s'est ainsi tenu le 14 octobre 2016 à la Maison de la Recherche à Paris $^{1}$. Ont participé à ce colloque à la fois des spécialistes de Pascal et de Chestov, et des chercheurs pour qui la question de la foi et de la raison était essentielle. Les actes en ont été publiés un an plus tard dans le numéro 2017 des Cahiers Léon Chestov. Tant nombre de questions qui y sont abordées se situent au cœur de la pensée pascalienne, il n'a pas paru inutile de proposer un résumé de ces actes aux lecteurs du Courrier Blaise Pascal.

2 Dans sa conférence inaugurale, «Un pascalien au pays de Chestov ", Gérard Ferreyrolles" ${ }^{2}$ à partir de la découverte, qu'il qualifie d'heureuse, de La nuit de Gethsémani, Essai sur la philosophie de Pascal, analyse la manière dont son auteur a lu les Pensées. Dans un premier temps, il critique le caractère arbitraire de cette lecture, évoquant les exemples de la séparation, contredite par les données historiques, entre le Pascal des Provinciales et le Pascal des Pensées, l'oubli du bonheur du croyant, le statut de la raison chez Pascal réduite à sa dénonciation, la mise entre parenthèses du caractère dialectique de l'apologétique. Dans un second temps, Gérard Ferreyrolles n'en souligne pas moins l'aspect «profondément pascalien» du livre de Chestov, notamment par ses formules vigoureuses, par une même radicalité de la foi, la 
perception de l'enjeu de l'apologétique comme étant d'éternité, de salut. Il conclut en affirmant qu'il n'est pas illégitime de ne pas se limiter à « une lecture philologique et historique des Pensées ", que l'interprétation des Pensées n'est pas la propriété exclusive des "pascaliens professionnels", des "critiques littéraires». Finalement Chestov a recherché la Vérité, non la vérité d'un texte, et s'est tourné vers la Révélation en récusant la raison.

Ramona Fotiade 3 , à travers son étude "Foi et raison chez Léon Chestov et Blaise Pascal ", s'est attachée à faire ressortir les points saillants, et le plus souvent justes, de la lecture chestovienne de l'apologétique pascalienne à partir de la thématique du sommeil et de l'éveil. Lecteur des Pensées et des écrivains russes (notamment Dostoïevski), Chestov oppose vérités scientifiques et Révélation biblique. La vertu insigne des Pensées est de nous délivrer du sommeil provoqué en nous par les évidences de la raison, de nous éveiller à une autre réalité, cachée par l'habitude. Pascal nous apprend à chercher Dieu par-delà la raison. Ce serait en quittant la logique, la connaissance, l'éthique que l'on pourrait retrouver l'arbre de la vie. Pour la pensée existentielle de Chestov, la foi est préparation à la mort. Sensible à la réflexion de Pascal sur l'immortalité, comme lui Chestov s'étonne de l'indifférence commune des hommes devant leur anéantissement à venir et de leur attachement aux choses éphémères. Le but de la philosophie, qui est lutte, devient dès lors de briser l'assoupissement incompréhensible des hommes, de chercher le sens de la vie par-delà les limites et contraintes de la raison, d'éveiller à la vérité de la foi.

Dans « Léon Chestov et Blaise Pascal, lecteurs de l'Écriture. D'une foi sans raison aux raisons de la foi ", Bernard Grasset ${ }^{4}$ prend comme point principal de rencontre entre les deux penseurs leur rapport à la Bible. Outre son essai La nuit de Gethsémani, Chestov se réfère régulièrement à l'auteur des Pensées dont il se sent un héritier. Il comprend la pensée de Pascal comme une pensée angoissée, gémissante, abyssale, au centre de laquelle se trouve le récit du péché originel qui montre que l'irrationnel seul explique l'homme. Pascal serait un penseur de l'accès au divin par le cœur, un penseur de l'éclair, non de l'évidence comme Descartes, un philosophe mystique, un philosophe de la Bible en lutte contre la raison. Ignorant la place donnée à la raison par Pascal, fondatrice dans la science, limitée mais nécessaire dans la religion, et l'importance de l'éthique judéo-chrétienne dans les Pensées, Chestov pensera la foi comme absurde et par-delà bien et mal. Juif proche du protestantisme, Chestov, pour qui le sola fide ne se dissocie pas du sola scriptura, définira avec justesse les Pensées comme une « interprétation de la révélation biblique ». Pour Pascal et pour Chestov, à l'encontre de la philosophie dominante, la philosophie ne peut atteindre la vérité qu'en perdant son autonomie et en se tournant vers l'Écriture. Tout dépend chez eux de la foi qui devient source de renouvellement de la pensée. Chez Pascal la philosophie biblique serait une philosophie de la foi, appuyée sur la raison, chez Chestov une philosophie de la seule foi, sans raison.

5 Alexandre Broadie ${ }^{5}$, dans son article « Le rôle de la raison dans l'assentiment de la foi Pascal, Chestov et l'arrière-plan du Moyen-Åge tardif", distingue trois sortes d'assentiment : l'assentiment d'opinion, celui d'évidence et celui de foi. L'assentiment de foi, fruit d'un acte de la volonté à partir d'un argument jugé probable, se situerait entre l'opinion et l'évidence. La réflexion se tourne alors vers Pascal et Chestov à partir de trois assentiments de foi considérés comme archétypiques: l'agonie de Jésus au Jardin de Gethsémani, la ligature d'Isaac et le péché originel. Si la contemplation de 
Jésus en son agonie invite à quitter une vie de confort, il est impossible de rester éveillés jusqu'à la fin du monde comme le demande Pascal. Cet assentiment de foi n'est pas tiré d'un argument probable. De même pour la ligature d'Isaac : qu'un père puisse vouloir sacrifier son fils aimé est une absurdité. En ce qui concerne la doctrine du péché originel, selon laquelle tous les descendants d'Adam héritent de la culpabilité liée à sa désobéissance, elle est folie selon la loi de non-contradiction, une brèche dans la relation entre foi et raison. Mais pour Chestov comme pour Pascal, l'impossibilité d'un article de foi aux yeux de la raison n'implique pas pour autant qu'il ne soit pas vrai. En conclusion ne peut-on pas de demander si l'assentiment pascalien de foi aux grands mystères bibliques ne rejoint pas plus profondément notre nature humaine que la doctrine des derniers médiévaux?

6 Dans «L'arbre de la science et l'arbre de la vie - Chestov et Fondane, lecteurs de Kierkegaard ", Jean-François Petit ${ }^{6}$ souligne que, pour Chestov, la source lointaine de la philosophie de la raison se situe dans la préférence originelle de l'homme pour les fruits de l'arbre de la science et son oubli de l'arbre de la vie. La métaphysique n'admet plus comme vrai que ce que la raison considère comme vrai. L'homme ne pense plus que la vérité pourrait se trouver dans l'Écriture. Pour Chestov, attentif aux profondeurs mystérieuses de l'existence humaine, Kierkegaard est le philosophe qui s'oppose à la conciliation hégélienne de la raison et de la religion. Il nous enseigne à préférer la foi et l'expérience à la raison et la connaissance. À la différence de la philosophie spéculative, de la philosophie de la raison, la philosophie existentielle est une philosophie de la Révélation biblique qui n'ignore pas l'angoisse du néant, une philosophie de la foi. Pour Benjamin Fondane, seul vrai disciple de Chestov, la pensée de Kierkegaard est l'expression de la «conscience malheureuse ». Mystère rationnellement inexplicable demeure l'existence. Si Fondane ne dénie pas une utilité pratique à l'intelligence et l'éthique, il n'en pense pas moins avec Chestov, ce chercheur de Dieu pour qui la philosophie engageait tout l'être, que l'arbre de la science donne la mort et qu'il faut revenir à l'arbre de la vie.

7 Julia Schwartzmann ${ }^{7}$ consacre son intervention à « Une étude de l'irrationalisme: du Kuzari médiéval à la pensée religieuse de Léon Chestov». Poète et penseur juif médiéval, Yehuda Halevi (1075-1141) fait dialoguer dans le Kuzari, sous-titré Apologie de la religion méprisée, un rabbin et un roi païen en recherche de la "bonne " foi. Il y critique le rationalisme médiéval. Quant à Chestov (1866-1938), il se situe dans l'héritage de Tertullien pour qui Athènes (la raison) n'a rien à voir avec Jérusalem (la foi). Penseur religieux qui mêle judaïsme et christianisme et qui écrit dans un style répétitif, métaphorique, empreint de beauté, Chestov ne cesse d'attaquer les philosophes grecs et modernes coupables de tout ramener à la raison. Pour Halevi, si la foi est au-dessus de la raison, si la Révélation (la Torah) répond seule aux questions essentielles de l'homme, la philosophie peut être utile à ceux qui n'ont pas reçu la Révélation et le «credo» n'est pas le contrepoint inséparable de l'«absurdum ${ }^{8}$ ". Chestov se sent en grande affinité avec Pascal, avec son Dieu d'Abraham, d'Isaac et de Jacob, mais le contenu de sa foi est plus vague, imprécise, que chez ce dernier et Halevi. L'Écriture cache des mystères, alors que la connaissance, nous dit-il dans Athènes et Jérusalem, empoisonne la joie de l'existence. En résumé, Chestov pose les mêmes questions que celles du Kuzari : le rapport entre foi et raison, Dieu de l'Écriture et Dieu des philosophes, Révélation et philosophie ${ }^{9}$. Mais, radical, il rejette non seulement le 
rationalisme mais aussi la raison alors que Halevi, plus mesuré, rejette le rationalisme mais pense que la raison peut être utile pour comprendre la Révélation ${ }^{10}$.

Dernière étude des actes: "La liberté au principe» d'Andrea Bellantone ${ }^{11}$. Pour Chestov, le renouvellement de la philosophie passerait par l'accueil de la pensée biblique. Alors que la philosophie spéculative se fonderait sur la nécessité, la philosophie biblique se fonderait sur la liberté. En assumant la pensée biblique, Pascal opérerait une rupture avec la philosophie traditionnelle de la raison. À la suite de celuici, Chestov, constant défenseur d'une "philosophie religieuse », d'une "philosophie biblique ", entend réaliser un "saut » dans une pensée posant la liberté au principe, une pensée biblique, au-delà de la rationalité. Mais alors on pourra se demander : «à quoi bon la philosophie après la Bible ? "S'opposant à Chestov et à sa lecture de Pascal, A. Bellantone émet l'hypothèse que placer la liberté au principe n'exige pas nécessairement de recourir à la Révélation biblique. La raison peut se réformer et s'adapter pour, à la faveur d'un acte réflexif, prendre en compte notre expérience de la liberté, cette irruption de l'éternité dans le temps, et l'ériger en principe ${ }^{12}$. La raison pourrait s'élargir sans s'anéantir dans la foi biblique. Une telle philosophie, ni classique, ni théologique, peut entrer en dialogue avec la pensée biblique, la rencontrer.

Ainsi, à travers ce colloque, des thématiques tout à fait essentielles de la pensée de Pascal et de Chestov ont-elles pu être abordées sous des angles multiples, nouveaux et variés. La lecture de l'œuvre de Pascal par Chestov est souvent partiale, exprimant ses propres vues, mais toujours stimulante. Et la confrontation entre ces deux penseurs croyants de l'existence par des chercheurs d'horizon divers autour de l'inépuisable et fondamentale question des rapports entre foi et raison a pu esquisser des voies vers une position équilibrée de l'esprit entre irrationalisme et rationalisme.

\section{NOTES}

1. 2016 marquait le cent-cinquantenaire de la naissance du philosophe russe.

2. Professeur de littérature française $d u \mathrm{xVII}^{\mathrm{e}}$ siècle, Université Paris Sorbonne Paris IV.

3. Présidente de la Société d'Études Léon Chestov, maître de conférences en littérature française, Université de Glasgow.

4. Chercheur associé, UCO Angers.

5. Professeur de philosophie, Université de Glasgow.

6. Maître de conférences en philosophie, Institut catholique de Paris.

7. Professeur de philosophie, Western Galilee College, Acre.

8. «C'est croyable parce que c'est absurde» soulignait Tertullien, affirmation souvent reprise par Chestov sous sa forme apocryphe plus connue : "Credo quia absurdum ».

9. Autant de questions aussi éminemment pascaliennes. 
10. En cela Pascal est plus proche du second que du premier.

11. Doyen de la Faculté de philosophie, Institut catholique de Toulouse.

12. Le conférencier parlera à ce sujet d'éleuthériologie (ou métaphysique de la liberté).

INDEX

Mots-clés : Pascal, Chestov (Léon), foi, raison.

Keywords : Pascal, Chestov (Léon), faith, mind

\section{AUTEUR}

BERNARD GRASSET

Chercheur associé à l'Université catholoque de l'Ouest, Angers. 